\title{
Innovation in the ICT infrastructure as a key factor in enhancing road safety. A multi-sectoral approach
}

\author{
Francesco Losurdo, Ivano Dileo \\ Department of Political Science \\ University of Bari Aldo Moro \\ Bari, Italy \\ francesco.1osurdo@uniba.it,ivano.dileo@uniba.it
}

\author{
Mirosław Siergiejczyk, Karolina Krzykowska \\ Faculty of Transport \\ Warsaw University of Technology \\ Warsaw, Poland \\ msi@wt.pw.edu.pl, kkrzykowska@wt.pw.edu.pl
}

\author{
Michał Krzykowski \\ Faculty of Law \\ University of Warmia and Mazury in Olsztyn \\ Olsztyn, Poland \\ m.krzykowski@onet.eu
}

\begin{abstract}
The ITSs (Intelligent Transportation Systems) are part of the ICT infrastructure and are built taking into account numerous legal and organisational conditions and individual needs of the users. They are the answer for wide requirements due to implementation of innovations in road transport. The primary objective of using ITSs is to improve the efficiency of traffic and traffic safety. The aim of this paper is to present a technical approach aiming to model the impact of environmental conditions such as urban traffic, visibility, other weather conditions and road conditions on road traffic safety, using the fuzzy logic and trying to explain also the economic and legal determinants of road safety as "non technical" parts.
\end{abstract}

Keywords- innnovation, ITS, safety of road transport

\section{INTRODUCTION}

Telematics combines elements of automatics, informatics and telecommunication. Thanks to such a combination telematics is becoming a basic technicality having its application into the modern transport system. This is a spread opinion, but it generally does not take into consideration any additional contribution - apparently quite different- coming from other scientific fields. In fact, the modern transportation system is more and more complex as skills and specialisation that even took no part in technical process, at any rate contribute to solve warmly felt problems [16]. One of these issues is the safety of transport that, one at a time, is the outcome of a combination of technicalities and humanities $[22,25]$.

Currently, innovations in telematics constitute the basis for Intelligent Transportation Systems (ITSs) and their provided services, which are implemented worldwide. These innovations try to give an answer to the increasing demand for road safety, ITSs and high level design architecture [24].

In fact, while the transport modalities, out of the road one, have devoted routes managed by telematics, the road transport system is not supported by telematics yet and the management is entrusted to the individual ability, sensations and reactions. Then, an ITS applied to the road safety is more complex because it must connect a lot of decision makers such as drivers, pedestrians and others who can make different decisions and behaviours. In addition, the safety system has to control the techniques and materials of construction of roads, the cars engineering, power and performance, the system of penalties and the maintenance of road [4].

Consequently, the concept of road transport safety is continuously widening, involving not only the standards of technical materials but also the behaviour of people and the conditions of the environmental context. This involves economic variables and legal regulations: economics and law are charged to face a big challenge to reach higher level of safety, mostly depending on "non technical" linkages.

In this paper, authors tried to look at the ITS applied to the road safety from the point of view of telematics, economics and law, matching different expertises to implement and manage a Road Intelligent Transport System-RITS. The main goal is to answer to the goals planned by the European Union Road Safety Programme 2011-2020 (MEMO/10/343, Brussels, 20 July 2010) and the related application by its member states.

The paper is organised as follows. Firstly, it investigates the "non technical" factors affecting the road safety, such as economic and legal elements connected with the management of safety in the frame of a road network; then, it introduces a fuzzy model in order to stylise the impact of the apparently "external" conditions on road traffic safety viewed through a technical approach directly to look at the environmental conditions such as urban traffic, visibility, other weather conditions and road conditions. Furthermore, it presents the requirements for ITS design and, finally, the conclusions. 


\section{STATE OF ART}

Development of ITSs and growth of the level of safety in the transport network are generally due to the application of telematics into the air, sea and rail transport systems while road transport traditionally played a secondary role. Probably, the road transport was considered self-regulated sector thanks to the human risk management capacity and the upgrading of the automative apparatus and the toolbox in the road transport vehicles.

The same European Road Safety Programme 20102020 shows the above point of view: general goal is the reduction of number of accidents, the death and injured people events; the priority is to focus on the quality of materials, building technologies of roads and so on, while the telematics is not included into the toolbox of road safety.

Subsequently, this traditional view of the above Programme looks at the economic sustainability and regulation to assure the road transport safety, that remains a secondary goal. It is evident that the road transport system safety is not so easy to manage if it is not supported by ICT and telematic techniques.

Thus, could the road safety do it without new technologies of information and communication in real time? At any rent, the guideline of European sectoral plan is not so poor: it puts on the desk the problem, defines the goals and lets the Member states design the ways to implement the same programme.

The application of Road Safety Programme at the domestic and regional level catched the EU guidelines and implemented them through ICT and telematics toolbox also to be consistent with innovative outcomes of automative and apparatus of vehicles.

Finally, the European Programme is consistent with the general planning methodology of EU: it lays down the main goals and address, and lets Member states transfer these last points within the implementing projects. Such method is correct and coherent because:

a) it is based on the matching of the top-down and bottom-up planning methodology;

b) it takes in the due account the conditions of road transport systems at local level;

c) it considers the true territorial context and the connected variables;

d) it stimulates the capacity of designing at the different levels of government and management.

The experiences are not so many as we can see from the above frame; neverthless, their rendering shows that Programme's guidelines are correctly perceived and applied.

\section{INNOVATION AND ITS}

Since Joseph Schumpeter's seminal work (1939), the characteristics of innovation processes and the introduction of a new product were considered as determinant of growth and development. Subsequently, Porter has also considered innovation as a process able to increase market competitiveness [17].

To date, it is widely accepted that innovation and technological upgrading are multidimensional processes based on a broader dimension involving many factors and areas $[9,18]$. Consequently, the changing nature of new technologies, due to the challenges launched by the global value chain, leads to new patterns of technological upgrading.

Thus, innovation may introduce new ideas, i.e. new industrial products, new process of production, new services characterised by higher knowledge levels [8].

It follows that innovation and technological upgrading are not only technical processes but also complex economic, social, political, legal aspects depending on knowledge and the channels in which individuals exchange, transfer and apply information through interactive learning systems.

Particularly, in the field of transport the concept of innovation and technological upgrading is mostly dependent on the use and quality of the Information and Communication Technology (ICT) considered as a tool useful to enable more efficient operations in transport supply chain [5].

Thus, the concept of "intelligent transport" has been recently used to introduce advantaged communication technologies useful for the upgrading in transport system and for the future management of the whole supply chain, involving road safety system $[13,15]$.

Nevertheless, converting transport infrastructures into intelligent transport system is not an easy task and requires wider investments and resources especially where the innovation level is weak or inside transition economies.

Furthermore, we have to also consider the diverse technological trajectories experienced by territories and related institutional context in which firms system and public operators are located. It follows that, wider financial resources are necessary to renovate physical and technological infrastructures as well as digital systems.

Particularly, lot of financial and technological resources are devoted to the safety and transport networks' control, mostly air, sea and rail transport; conversely, road transport safety has been generally entrusted to a simpler technology and management. Consequently, new applied business models are necessary to facilitate the incorporation of innovation and then to fasten the convertion of a transport system into an intelligent transport system.

\section{ECONOMIC CONSIDERATIONS ON ROAD TRANSPORT SAFETY}

In the convertion process into intelligent transportation system also "non-technical" elements have to be considered. Firstly, economic variables connected to the innovation production, technology transfer, all useful into strategic fields (such as transport safety and control): these variables are much more crucial if we consider transport networks formed by different parts and several technical modalities.

At the same time, the modern road transport system is global. Traditionally, the roads were viewed as material infrastructures connected with the construction of automobile industries, because of their wide sectoral interdependence. Also in the economic literature the impact of road construction was considered the main factor able to invert the depressive cycle phase and to sustain the economic growth [1]

Accordingly, in the vision of a global economic system, the road transport infrastructure remains a strong 
factor of growth, both at local dimension [19] and at a global level [2].

Moreover, road transport system is not only a network for moving people and freight, but also a part of whole and so an integrated connectivity system based on undefined borders. This means that the transport network is becoming more complex because of the extension and specialisation of demand of road transport services and the growth of transported freight, such as liquids, gas, waste, etc.; then, the de visu control cannot be able to control such complexity [14] requesting higher standards of safety.

Under the above conditions of safety demand pressure, the application of ICT tools -firstly telematics- is necessary, because they are:

1. able to manage long-distance connections;

2. useful to prevent the risk situations connected with road transport;

3. cheaper and more efficient than the human control.

Consequently, the non-material components of the road networking are growing up more than the material ones because they are considered suitable to solve the related safety problems. Moreover, the main mission of Internet of Things (IoT) is to create a "digital environment" in which it is included the road transport safety as a part of a general "Transport Digital Ecosystem", based on telematics and other ICT.

In fact, the risky condition may also effect the competitiveness between operators that request different road transport services in terms of people and goods mobility that can be regulated by the application of ITS.

Furthermore, the ITS includes applied researches, innovations, technology transfer and the connected services of sperimentation and demonstration; finally, the instrumental production of hardware, applied software, regulation and control sytems and so on that are the structural elements of the Transport Digital Ecosystem.

\section{LAW REGULATIONS FOR IMPLEMENTING ITS SYSTEMS}

The second non technical element is represented by the legal conditions of transport. There is a number of legal regulations relating to the implementation of the described service:

- Directive of the European Parliament and of the Council 2010/40/EU of 7 July 2010 on the framework for the deployment of Intelligent Transport Systems in the field of road transport and for interfaces with other modes of transport, in particular regarding compliance with the rules on specifications and deployment of ITSs indicated in Annex II to the said Directive;

- Commission Delegated Regulation (EU) No 886/2013 of 15 May 2013 supplementing Directive of the European Parliament and of the Council 2010/40/EU with regard to the data and procedures for free transfer of, if possible, minimum general information about traffic related to road safety;

- Commission Delegated Regulation (EU) of 18 December 2014 supplementing Directive of the European Parliament and of the Council 2010/40/EU with regard to provision of general EU information services in real-time traffic concerning traffic;
- Act of 20 June 1997 - the Traffic Law Act Journal of Law of 2012, item 1137, as amended;

- Act of 21 March 1985 on public roads Journal of Law of 2013, item, as amended;

- Act of 17 February 2005 on informatisation of the activity of entities that perform public tasks Journal of Law of 2013, item 235, as amended;

- Resolution of the Council of Ministers of 12 April 2012 on the National Interoperability Framework, minimum requirements for public registers and exchange of information in an electronic form and minimum requirements for ICT systems;

- Resolution of the Minister of Infrastructure of 23 September 2003 on the detailed terms of traffic management on roads and supervision over this management Journal of Laws No. 177, item 1729;

- Resolution of the Ministers of Infrastructure and the Interior and Administration of 31 July 2002 on road signs and signals. Journal of Laws of 2002, No. 170, item 1393;

- Resolution of the Minister of Infrastructure of 3 July 2003 on the detailed technical requirements for road signs and signals and traffic safety devices and conditions of their placement on roads Journal of Laws of 2003, No. 220, item 2181, as amended;

- $\S 114$ para. 1 of the resolution of the Minister of Transport and Maritime Economy of 2 March 1999 on the technical conditions, which public roads and their location should meet (Journal of Laws of 1999, No. 43, item 430).

Other EU legal acts in the field of ITS, e.g.: ISO TS 15624 Transport information and control systems - Traffic Impediment Warning System (TIWS) - System requirements.

\section{MODELLING THE IMPACT OF EXTERNAL CONDITIONS ON ROAD TRAFFIC SAFETY}

It may be observed that implementing intelligent transportation systems is usually done after wide researches including environmental analysis. In fact, there are a few external conditions which directly affect the road safety, such as dimension of urban traffic, visibility on the road, meteorological conditions or road condition. A model of those factors affecting road traffic safety can be done by using fuzzy logic sets [21].

Fuzzy set is a mathematical object with a defined membership function which takes values in the range $[0$, 1]. The theory of fuzzy sets was introduced by Lotfi A. Zadeh in 1965 as an extension of the classical theory. This reasoning is applied in case of inaccurate or incomplete information, which makes it necessary to take decisions under conditions of uncertainty. Knowledge of the phenomenon is subjective and is expressed by the opinion or descriptive expert studies. It is determined by an informal basis as linguistic uncertainty. Their big advantage is possibility to graphically present the results of research, model uncertainties and do researches considering the effect of multiple factors on different phenomenon [3].

In research works, it is planned to use the Mamdani fuzzy model. This type of model, to solve the problem, uses a linguistic approach, as well as the fuzzy conditional 
sentences and an assembly inference rule. In the process of building the model, two stages should be distinguished:

1 indication of the input variables and definition of their terms (based on the literature research);

2 assigning ranges of variable linguistic values.

It is extremely important to assign membership function to each of the variables. Its task, in fuzzy reasoning, is to express the extent, to which each element $x$ of the universe of discourse $X=\{x\}$ belongs to the fuzzy set $\mathrm{A}-$ from $\mu \_\mathrm{A}(\mathrm{x})=0$ for total non-membership to $\mu$ A $(\mathrm{x})=1$ for full membership though all intermediate degrees $\left(0<\mu \_A(x)<1\right)$.

After analysis of the existing literature, MatLab's Fuzzy Logic Tool was chosen, in which a gauss function was applied to describe the input data. Currently, four groups of external factors which are input data have been distinguished in the model, including: urban traffic, visibility on the road, meteorological conditions or road condition. Then, 81 rules specifying dependencies between particular factors in the road traffic safety were set.

TABLE I. LINGUISTIC VALUES OF INPUT AND OUTPUT VARIABLES RANGES

\begin{tabular}{|c|c|c|c|}
\hline I/O variable & \multicolumn{3}{|c|}{ Linguistic values } \\
\hline Urban traffic & small & medium & big \\
\hline Visibility & low & medium & good \\
\hline Meteo conditions & bad & medium & good \\
\hline Road condition & bad & medium & good \\
\hline $\begin{array}{c}\text { Impact on road } \\
\text { traffic safety }\end{array}$ & low & medium & high \\
\hline
\end{tabular}

The standard linguistic values in the range from large positive to large negative were introduced into the model. The rule base was built on the basis of the analysis of the literature and available results of research conducted in the similar field.

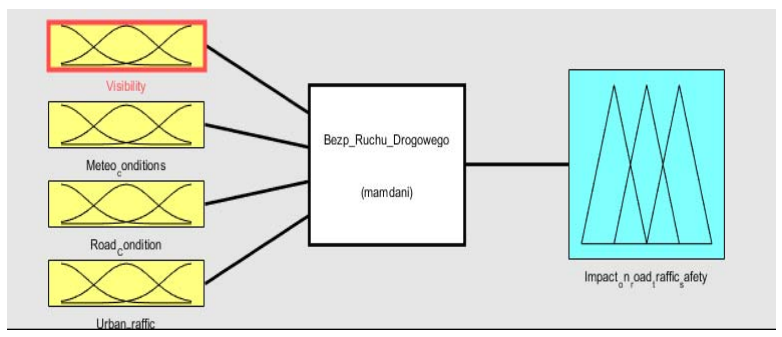

Figure 1. Mamdani model to simulate impact of external conditions on road traffic safety

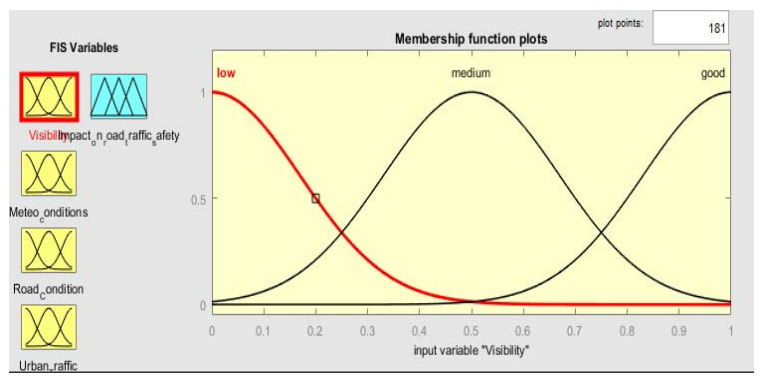

Figure 2. Adopted membership functions for fuzzy sets describing an input datum: visibility
As an example, Figure 2 shows the adopted membership functions for fuzzy sets describing an input datum: visibility (according to Table 1). In the further part of the description, the graphic interpretation, which define the relationships between the particular external factors and their influence on road traffic safety, was presented.

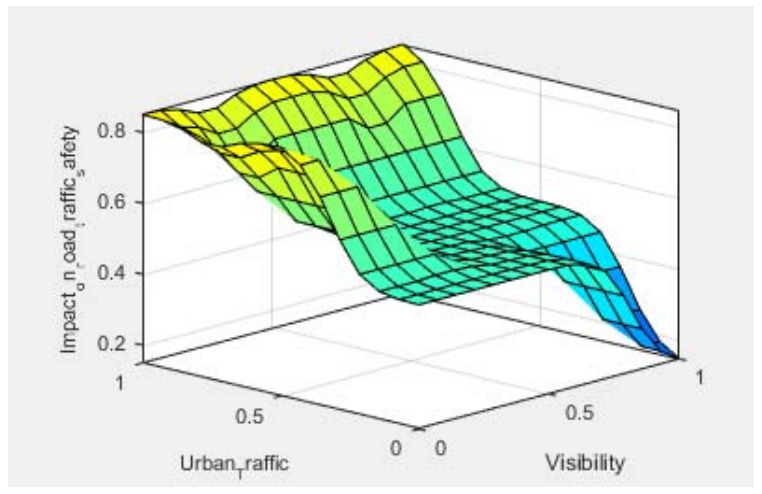

Figure 3. Graphic results for impact on road traffic safety depending on urban traffic and visibility

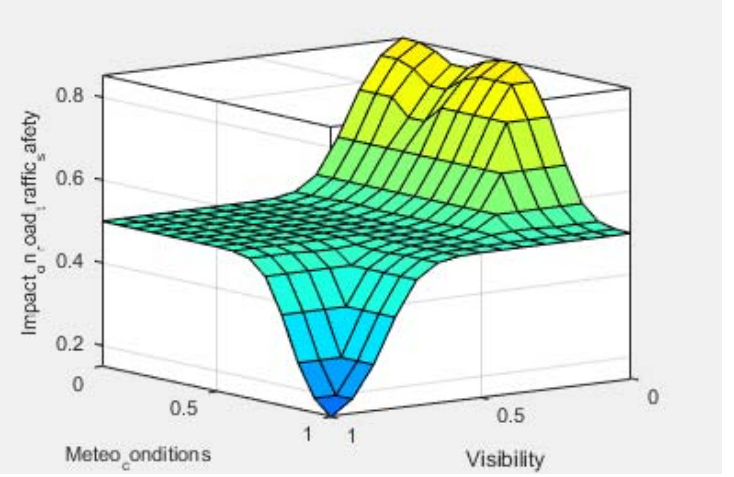

Figure 4. Graphic results for impact on road traffic safety depending on meteo conditions and visibility

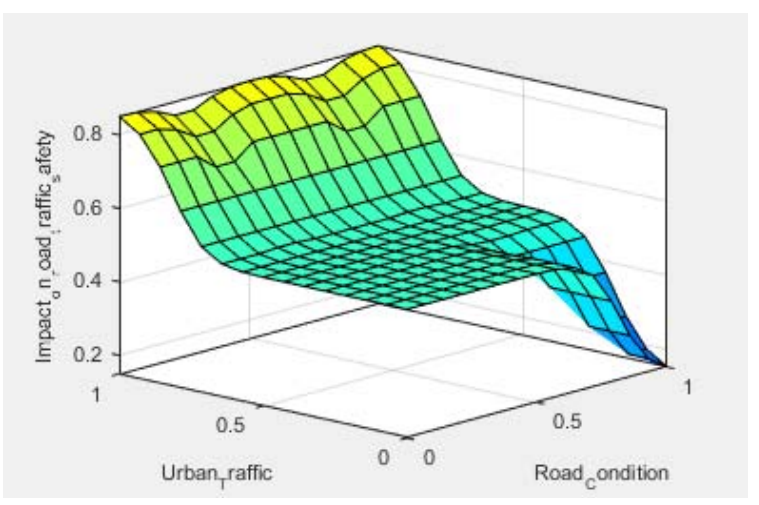

Figure 5. Graphic results for impact on road traffic safety depending on urban traffic and road condition

In the Figure 3, one can clearly observe that the lower visibility and the bigger urban traffic the higher is their 
impact on road traffic safety. It translates into a large number of accidents. Similarly, the lower visibility and the worse meteorological conditions - also the higher impact on safety on the road (Fig. 4). The graph on Fig. 5 is a premise to say that big urban traffic is a more important factor than road condition which impacts safety in a rather medium level. Therefore, an impact of external conditions on road traffic safety is proven. Preliminary tests conducted for the needs of this work confirm this fact.

\section{REQUIREMENTS FOR ITS DESIGN}

As we proved the impact of external conditions on road traffic safety we may now suggest a solution excluding it. There is an expanded list of ITS services and systems. The subject of interest of this paper can involve ITS, which provides the traffic monitoring and controlling service $[6,7]$. Therefore, it is important to present the requirements for designing of such a service, which includes, among others:

- traffic monitoring;

- CCTV system;

- monitoring of weather conditions;

- management of the road maintenance, that is, monitoring of the road pavement condition.

The traffic monitoring involves the collection of traffic data from detectors connected to the controllers [10]. The vehicle detection is carried out with the use of inductive control and classification loops. The classification detectors are installed on all traffic lanes on the inlets and outlets of intersections covered by the system. In order to detect the presence of pedestrians and cyclists, the buttons on the right side of pedestrian crossings or passages are used.

CCTV system is to carry out the following tasks:

- ongoing monitoring of road traffic;

- CCTV system recording of the operation of traffic lights;

- monitoring of traffic incidents and road accidents;

- data archiving.

The service should be designed in an open way that can be adapted to new conditions, which is to facilitate the system future development [12]. The monitoring should include all intersections covered by the traffic control system. It should be possible to have a preview from cameras in the traffic control centre together with the data archiving in high quality for a minimum period of 1 month, on the dedicated recorders. The main centre of the monitoring system should be located in the Traffic Control Centre. The application for viewing the monitoring image is available in all stations of the Traffic Control Centre.

In turn, the service which allows to monitor the weather conditions, is to implement the following tasks:

- direct measurement of meteorological parameters (determination of the current state of meteorological conditions);

- provision of information on the current state of meteorological conditions.

The system collects and gathers the following data from the road meteorological stations:

- air temperature,

- road surface temperature

- relative air humidity,

- dew point,
- wind direction and speed,

- $\quad$ precipitation type.

The service of monitoring the road pavement condition involves the monitoring of the road pavement condition and its environment in real time. The system generates messages for the operator, which are related to the possibility of the occurrence of black ice and meteorological hazards for road traffic. All the data collected by the road measurement stations are accumulated in the database for the minimum period of a year. It is important to provide software for the interpretation, reporting and creation of graphs with all the collected data. The data transmission from the road meteorological stations is provided with a frequency of at least every minute [23].

Such a complex solution of ITS system may be the answer to both external conditions influencing road traffic safety and a need for innovation in this area including legal requirements.

\section{CONCLUDING REMARKS}

The provision of taking decisions based on reliable data and knowledge on current and expected weather conditions contributes to the provision of the traffic flow, and thus, the improvement of the road safety level. The ongoing monitoring of traffic allows to quickly detect an incident, respond to it, and minimise its effects.

The ITSs are built taking into account numerous legal and organisational conditions and individual needs of the users. Therefore, they are not finished products and their implementation requires a clear definition of functional and technical requirements and, ultimately, the description of the procurement subject used in connection with public procurement procedures. The primary objective of using ITSs is to improve the efficiency of traffic and traffic safety (i.e. reducing the number of traffic accidents, reducing the severity of accidents in the form of the reduction of fatalities, the seriously injured and the injured, or the number of accidents involving unprotected traffic participants - motorcyclists, cyclists and pedestrians). The aim of the paper was to present the importance of introducing multidisciplinary approach in the road transport safety planning and governance. It seems definite that the needful innovations in road safety system are:

- An ITS based on telematics which may reduce the impact of external conditions on road traffic safety within a general "Transport Digital Ecosystem";

- the use of friendly techniques like Internet of Things (IoT);

- the creation of a "digital environment" in which IoT could be easily used.

\section{ACKNOWLEDGMENT}

The publication was prepared within the framework of the project of Development of Road Innovation (RID) funded by the National Centre for Research and Development and the Directorate for National Roads and Motorways (Contract No. DZP/RID-I-41/7/NCBR/2016). 


\section{REFERENCES}

[1] Barro R.J., Sala-i-Martin X. 1995. Economic Growth. New York, McGraw Hill.

[2] Bordo M.D., Taylor A.M., Williamson J.G. (Eds.). 2003. Globalization In Historical Perspective. Chicago, The University Of Chicago Press.

[3] Chen, T. 2012. A collaborative fuzzy-neural approach for long-term load forecasting in Taiwan. Comput. Ind. Eng. 63, 663-670.

[4] Chowdhury M. A., Sadek A. 2003. Fundamentals of Intelligent Transportation Systems Planning. Artech House ITS Library. Boston, London.

[5] Coronado Mondragon A.E, Lalwani C.S, Coronado Mondragon E.S., Coronado Mondragon C.E., Pawar K.S. 2012. Intelligent transport systems in multimodal logistics: A case of role and contribution through wireless vehicular networks in a sea port location', International Journal of Production Economics, Vol. 137 No.1, pp. 165- 175.

[6] Ehlers U.C \& Ryeng E.O. \& McCormack E. \& Khan F. \& Ehlers S. 2017. Assessing the safety effects of cooperative intelligent transport systems: A bowtie analysis approach. Accident Analysis \& Prevention. Volume 99, Part A, pp. 125-141.

[7] Fries, R. \& Chowdhury, M. \& Brummond, J. 2009. Transportation infrastructure security utilizing intelligent transportation systems. New Jersey: John Wiley $\square$ Sons.

[8] García-Pereiro T. \& Dileo I. 2015. Determinants of nascent entrepreneurial activities: the Italian case. RIVISTA ITALIANA DI ECONOMIA DEMOGRAFIA E STATISTICA. VOLUME LXIX - N. 4. Roma, pp. 5-16.

[9] Jindra, B., Dominguez Lacasa, I., Radosevic, S. 2015. Dynamics of Technology Upgrading of the Central and East European Countries in a Comparative Perspective: Analysis Based on Patent Data, Economics and Business Working Paper No.135, UCL SSEES Centre for Comparative Economics, February 2015.

[10] Klein L.A. 2001. Sensor Technologies and date requirements of ITS." Artech Hause, ITS Library.

[11] Krivolapova O. 2017. Algorithm for Risk Assessment in the Introduction of Intelligent Transport Systems Facilities. Transportation Research Procedia. Volume 20, pp. 373377.

[12] Łubkowski, P. \& Laskowski, D. 2015. Selected issues of reliable identification of object in transport systems using video monitoring services. In Communication in Computer and Information Science, vol. 471: 59-68. Berlin Heidelberg: Springer.

[13] Lumsden K., Stefansson G. 2007. Smart freight to enhance control of freight transportation networks, International Journal of Logistics Systems Management, Vol. 3 No. 3, pp. 315-329.

[14] Mandula L., Senn L. 2011. Managing the complexity: decision making process on sustainable mobility, available at ssrn:https//ssrn.com/abstract=2051455 or http://dx.doi.org/10.2139/ssrn.2051455.

[15] Manzie C., Watson H., Halgamuge S. 2007. Fuel economy for urban driving: Hybrid vs. intelligent vehicles, Transportation Research Part C, Vol. 15 No. 1, pp.1-16.

[16] Piro G., Cianci I., Grieco L.A., Boggia G., Camarda P. 2014. Information centric services in Smart Cities. Journal of Systems and Software (88). Elseview, pp. 169-188.

[17] Porter M.E., 1990, The Competitive Advantage of Nations. Free Press. ISBN 0-684-84147-9.

[18] Radosevic, S. and Yoruk, E. 2014. Are there global shifts in world science base? Analysis of catching up and falling behind of world regions, Scientometrics, Vol. 101, pp. 1897-1924.

[19] Paul Romer, 1994. New Goods, Old Theory, and the Welfare Costs of Trade Restrictions. Journal of Development Economics, 43(1): 5-38.
[20] Schumpeter J. 1939. Business Cycles: A Theoretical, Historical, and Statistical Analysis of the Capitalist Process. London: McGraw-Hill.

[21] Skorupski J. \& Uchroński P. 2016. A fuzzy system to support the configuration of baggage screening devices at an airport. Expert Systems With Applications 44, pp. 114125.

[22] Stawowy, M. \& Kasprzyk, Z. 2015. Identifying and simulation of status of an ICT system using rough sets, In: W. Zamojski (ed.), New Results in Dependability and Computer Systems. Proceedings of the 8th International Conference on Dependability and Complex Systems DepCoS-RELCOMEX: 477-487, Advances in Intelligent Systems and Computing, vol. 224. Springer.

[23] Sumiła M. 2012. Selected aspects of message transmission management in ITS systems. International Conference on Transport Systems Telematics. Springer Berlin Heidelberg, pp. 141-147.

[24] Siergiejczyk M., Rosiński A., Dziula P., Krzykowska K. 2015. Reliability-Exploitation Analysis of Highway Transport Telematics Systems. Journal of KONBIN (33), pp. 177-186.

[25] Zając M., Świeboda J. 2015. Process hazard analysis of the selected process in intermodal transport. International Conference on Military Technologies (ICMT). IEEE, pp. 1- 